\title{
Post-cardiotomy Acute Right Ventricular Failure: VA-ECMO Followed by Percutaneous Right Ventricular Assist Device with Tandem Protek Duo for a Successful Outcome
}

\author{
Wael Awad ${ }^{1}$, Joy Edlin ${ }^{1}$, Alina Mistirian², and Amman Sarang ${ }^{3}$ \\ ${ }^{1}$ St Bartholomew's Hospital \\ ${ }^{2}$ Affiliation not available \\ ${ }^{3}$ Barts Health NHS Trust
}

June 22, 2020

\begin{abstract}
A 60-year-old lady with a past history of rheumatic mitral stenosis (MS), percutaneous valvuloplasty and persistent atrial fibrillation represented with heart failure. Cardiac ECHO confirmed severe MS, moderate tricuspid and aortic valve regurgitation and pulmonary hypertension. She underwent mitral and aortic valve replacements, a tricuspid valve repair and left atrial appendage clip. She developed a LV wall rupture and underwent repair of defect. Cardiogenic shock persisted despite IABP counter-pulsation and escalating inotropic support. Central veno-arterial extracorporeal membrane oxygenation (VA-ECMO) and 9 days later, a percutaneous Protek Duo right ventricular assist device (RVAD) for RV failure afforded a successful outcome.
\end{abstract}

\section{Case Report:}

A 60-year-old lady presented with worsening dyspnoea (NYHA III), 3-pillow orthopnoea and peripheral oedema. Echo showed severe mitral stenosis (MS) and tricuspid regurgitation, moderate aortic regurgitation and a raised pulmonary artery systolic pressure of $50 \mathrm{mmHg}$ with preserved biventricular function. Her past medical history included rheumatic MS, requiring percutaneous valvuloplasty 15 years previously, and persistent atrial fibrillation. Her coronary angiogram showed unobstructed coronary arteries. Her Euroscore II was $8 \%$. She was referred for surgery.

At surgery, a rheumatic mitral valve (MV) with severe MS and almost obliterated chordae were confirmed. The papillary muscles were attached to the valve leaflets and had to be partly excised with the chords in order to make room in the left ventricular outflow tract. The patient received mechanical mitral $(27 / 29$ $\mathrm{mm}$ OnX) and aortic (21mm OnX, Cryolife) valve replacements, a tricuspid valve annuloplasty ring (28mm MC3, Edwards) and left atrial appendage clip (50 mm, AtriCure).

Cardiopulmonary bypass (CPB) was weaned with milrinone and noradrenaline infusions. Protamine was administered slowly, but the blood pressure dropped and was unresponsive to fluid resuscitation and adrenaline boluses. Vasopressin had no effect and methylene blue was given as a last resort, eventually raising the blood pressure.

Prior to closing the sternum, blood collected at the back of the heart. A small hole was found $2 \mathrm{~cm}$ away from the atrioventricular groove. $\mathrm{CPB}$ was re-instated and the defect repaired.

The right ventricle (RV) was now dilated and severely impaired with a CVP of $18 \mathrm{mmHg}$ and the patient was difficult to ventilate. Despite good left ventricle contractility, the patient required increasing inotropic 
support. When cardiogenic shock persisted, intra-aortic balloon pump (IABP) counter-pulsation was commenced with no effect. The patient was placed on central veno-arterial extracorporeal membrane oxygenation (VA-ECMO) with the chest left open and transferred to the intensive care unit.

RV failure with raised CVP and worsening respiratory function persisted. On post-operative day 9, a Protek Duo right ventricular assist device (RVAD; Protek Duo (TandemLife, Pittsburgh, PA)) was inserted percutaneously via right internal jugular venous (IJV) access, with the proximal inflow lumen positioned in the right atrium and the distal outflow lumen positioned in the main pulmonary artery (Fig. 1). An oxygenator was placed in the circuit. VA-ECMO was discontinued, the patient decannulated and the chest was closed.

By day 14 all support was weaned and the RVAD removed. The patient was extubated with no neurological consequences, but developed a ventilator-associated pneumonia and required a tracheostomy for seven days. She was discharged to her local hospital for convalescence and then home. She was well when reviewed as an out-patient 2 and 6 months later.

\section{Discussion:}

In our case, excision of the MV apparatus predisposed to the LV rupture - a recognised complication following MV replacement, although its incidence has reduced with sparing of the native valve. The prolonged procedure and $\mathrm{CPB}$ time resulted in RV failure.

Acute RV failure is a well-recognized cause of morbidity and mortality following cardiac surgery. It is seen more often after left ventricular assist device implantation (20-30\%), heart transplantation (2-3\%) and only in $0.04-0.1 \%$ of patients following cardiac surgery. ${ }^{1}$

Patients with acute RV failure post-cardiotomy have a poor prognosis. In a series of 30 patients undergoing RVAD implantation for isolated RV failure following cardiac surgery over an 11-year period to 2012, 13 patients were successfully weaned from RVAD and of these, 10 survived to discharge. The median duration of support was 5 days. $^{2}$

There have been 2 reports of insertion of an implantable RVAD following post-cardiotomy RV failure with successful weaning from the RVAD and explantation at 15 and 79 days of support, respectively. ${ }^{3,4}$ One patient developed a stroke and both needed redo sternotomy and surgery, which in itself is also associated with increased risk.

VA-ECMO was used initially as a bridge to recovery or decision in our unstable patient. ECMO provides adequate cardiopulmonary support in some instances but does not unload the ventricles to the degree possible with a ventricular assist device.

To our knowledge, this is the first report of the successful use of a percutaneous Protek Duo RVAD postcardiotomy. The Protek Duo RVAD is a good option for short-term RV support in isolation or with other LV support devices for bi-ventricular support. However, it requires access to a hybrid theatre and Cardiology support, as well as input from cardiac surgeons and intensivists and was felt to be inappropriate as a first-line treatment. Percutaneously placed with IJV access and with an oxygenator added to the circuit, it provides both RV and respiratory support, and allows patient mobility and rehabilitation while on support.

This case illustrates that VA-ECMO can act as an effective short-term bridge to recovery or further support with a percutaneous RVAD. The case also shows that early and aggressive treatment of RV failure can have a positive outcome and this strategy should be considered in selected patients.

\section{Conflicts of Interest:}

None declared.

\section{References:}


1. Haddad F, Couture P, Tousignant C, Denault AY. The right ventricle in cardiac surgery, a perioperative perspective: II. Pathophysiology, clinical importance, and management. Anesth Analg. 2009;108:42233.

2. Moazami N, Pasque MK, Moon MR, Herren RL, Bailey MS, Lawton JS, Damiano RJ Jr. Mechanical support for isolated right ventricular failure in patients after cardiotomy. J Heart Lung Transplant. 2004;23:1371-5.

3. Sertic F and Ali A. Acute-right-ventricular-failure post-cardiotomy: RVAD as a bridge to a successful recovery. J Surg Case Rep. 2018 Jun; 2018(6):rjy140.

4. Osaki S, Edwards NM, Johnson MR, Kohmoto T. A novel use of the implantable ventricular assist device for isolated right heart failure. Interact Cardiovasc Thorac Surg 2008;7:651-3.

\section{Figure legends:}

Fig. 1:

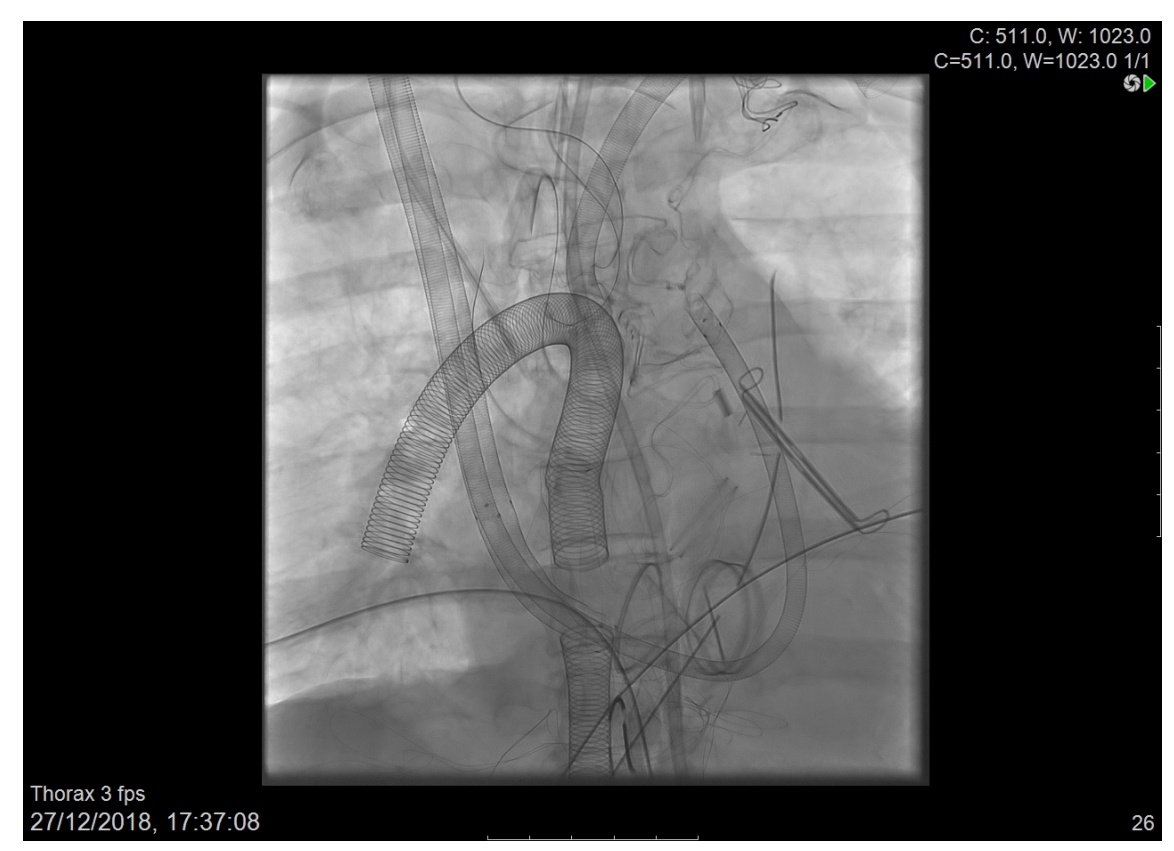

Figure 1. Chest Xray demonstrating (A) two-stage venous cannula and (B) aortic cannula of the VA-ECMO; (C) Tandem Protek Duo RVAD with its tip in the main Pulmonary Artery; (D) Aortic Valve Replacement; (E) Mitral Valve Replacement; (F) tricuspid annuloplasty ring; (G) Left Atrial clip.

Fig. 1: Chest Xray demonstrating the Tandem Protek Duo Right Ventricular Assist Device (67/90 characters)

Remove this picture and insert a Chest Xray with the RVAD only ie after the VA ECMO was removed.

\section{Hosted file}

Fig 1 JCS.pptx available at https://authorea.com/users/332983/articles/461201-postcardiotomy-acute-right-ventricular-failure-va-ecmo-followed-by-percutaneous-rightventricular-assist-device-with-tandem-protek-duo-for-a-successful-outcome 